\title{
Actitudes, percepciones y factores asociados a la vacunación antigripal en los profesionales de atención primaria de Navarra, 2011-2012
}

\author{
Attitudes, perceptions and factors associated with influenza \\ vaccination among primary healthcare professionals \\ in Navarre, 2011-2012
}

\author{
I. Martínez-Baz ${ }^{1,2}$, J. Díaz-González ${ }^{1}$, M. Guevara ${ }^{1,2}$, D. Toledo ${ }^{2}$, A. Zabala ${ }^{1}$, \\ A. Domínguez ${ }^{2,3}$, J. Castilla ${ }^{1,2}$
}

\section{RESUMEN}

Fundamento. La vacuna de la gripe está indicada en los profesionales sanitarios para proteger su salud y la de sus pacientes. El objetivo fue describir la cobertura de vacunación antigripal en los profesionales de atención primaria durante la temporada 2011-12 en Navarra y estudiar los factores asociados a la vacunación.

Material y métodos. Se envió una encuesta anónima vía web a los profesionales de atención primaria en 2012. La encuesta recogió información sobre el estado de vacunación antigripal de las últimas temporadas, sobre las actitudes y conocimientos de la vacuna y datos sociodemográficos y profesionales.

Resultados. De 1.083 profesionales, 381 (35\%) completaron la encuesta: $54,3 \%$ profesionales de enfermería, 35,7\% médicos de familia y $10 \%$ pediatras. En la temporada $2011-12$ se habían vacunado el $56,2 \%$, sin cambios significativos con respecto a temporadas previas. El principal predictor de la vacunación fue el haberse vacunado en alguna temporada previa (odds ratio (OR): 20,87; intervalo de confianza (IC) 95\%: 8,98-48,48). Se vacunaron más los que tenían personas con factores de riesgo en la familia (OR: 1,72; IC 95\%: 1,05-2,84), los que estaban preocupados por enfermar (OR: 4,59; IC 95\%: 2,30-9,15) y por contagiar a sus pacientes (OR: 6,29; IC 95\%: 3,02-13,13). El haber participado en actividades formativas sobre gripe y vacunas no influyó en la decisión de vacunarse.

Conclusiones. La cobertura de vacunación entre los profesionales sanitarios de atención primaria de Navarra está por debajo de los niveles deseables. Se debe mejorar la implicación de los profesionales en la estrategia de prevención de la gripe.

Palabras clave. Vacunación antigripal. Atención Primaria. Profesionales sanitarios. Cobertura de vacunación.

\begin{abstract}
Background. The influenza vaccine is recommended for all healthcare workers to protect their own health and their patients. We aimed to describe the influenza vaccine coverage in primary healthcare professionals in the 2011-12 season in Navarre and to study the factors associated with this coverage.
\end{abstract}

Methods. An anonymous web-based survey was sent to primary healthcare professionals in 2012. The survey collected information on the influenza vaccination status for the four previous seasons, the attitudes towards and knowledge of the vaccine, and socio-demographic and professional data.

Results. Of 1,083 professionals, 381 (35\%) completed the survey: $54.3 \%$ nurses, $35.7 \%$ general practitioners and $10 \%$ paediatricians. In the 2011-12 season, $56.2 \%$ had received the influenza vaccine, without significant changes compared to previous seasons. The vaccination in any previous season was the main predictive factor for current vaccination (odds ratio (OR): 20.87; confidence interval (CI) 95\%: 8.98-48.48). The vaccine coverage was higher among those living with persons with risk factors for influenza (OR: 1.72; CI 95\%: 1.05-2.84), those worried about becoming ill (OR: 4.59; CI 95\%: 2.30-9.15), or infecting their patients (OR: 6.29; IC 95\%: 3.02-13.13). Participation in training activities on influenza or vaccines was not significantly associated with influenza vaccination.

Conclusions. Influenza vaccination coverage in primary healthcare professionals in Navarre is below desirable levels. A greater involvement of healthcare professionals is necessary in the influenza prevention strategy.

Key words. Influenza vaccination. Primary heath care. Healthcare professionals. Vaccination coverage.
1. Instituto de Salud Pública de Navarra, Pamplona, España

2. CIBER Epidemiología y Salud Pública (CIBERESP), España

3. Departamento de Salud Pública, Universidad de Barcelona, España

Recepción: 29 de abril de 2013

Aceptación provisional: 10 de mayo de 2013

Aceptación definitiva: 21 de mayo de 2013

\section{Correspondencia:}

Iván Martínez-Baz

Instituto de Salud Pública de Navarra

C/ Leyre 15

31003 Pamplona, España

E-mail: imartinba@navarra.es

Financiación: Ministerio de Sanidad, Servicios Sociales e Igualdad (EC11-302) e Instituto de Salud Carlos III (GR09/0028 y GR09/0030 y PS12/00087). 


\section{INTRODUCCIÓN}

La vacunación antigripal es la principal medida preventiva con la que contamos para hacer frente a la gripe y sus complicaciones $^{1,2}$. Existen varias razones que justifican la vacunación antigripal anual de los trabajadores sanitarios: proteger su salud, evitar la baja laboral por enfermedad durante los períodos de mayor demanda de los servicios de salud, y reducir la morbilidad y mortalidad entre sus pacientes ${ }^{3-7}$. La vacunación anual frente a la gripe está recomendada para todos los profesionales sanitarios que no tengan contraindicaciones con el fin de evitar riesgos para su propia salud y la de sus pacientes ${ }^{8,9}$. Sin embargo, en España las coberturas vacunales en profesionales sanitarios no suelen alcanzar el $50 \%{ }^{10-12}$.

Los profesionales sanitarios de atención primaria tienen un papel fundamental en la prevención, diagnóstico y tratamiento de la gripe. Durante las ondas estacionales atienden la mayor parte de las consultas médicas relacionadas con la gripe, y por ello, tienen mayor riesgo de adquirir la enfermedad y también de actuar como transmisores.

Varios estudios en profesionales sanitarios en el ámbito hospitalario han descrito la cobertura de vacunación frente a la gripe, así como los factores asociados, los conocimientos y las actitudes ${ }^{12-15}$. Sin embargo, pocos han evaluado estos aspectos en los trabajadores de atención primaria $^{16,17}$.

La red de atención primaria de Navarra está formada por unos 1.000 profesionales sanitarios, incluyendo personal de enfermería, médicos de familia y pediatras. La vacuna de la gripe se recomienda y se ofrece de forma gratuita a todos los trabajadores sanitarios.

El objetivo de este estudio fue conocer la cobertura de vacunación frente a la gripe en los profesionales sanitarios de atención primaria durante la temporada 2011-12 en Navarra, e identificar las percepciones y actitudes de los profesionales frente a la vacunación antigripal.

\section{MATERIAL Y MÉTODOS}

\section{Diseño y población de estudio}

Entre marzo y abril de 2012 se llevó a cabo un estudio transversal a través de una encuesta a los profesionales de atención primaria de siete comunidades autónomas (Andalucía, Castilla y León, Cataluña, Comunidad Valenciana, Madrid, Navarra y País Vasco). La encuesta contó con el apoyo de las direcciones de Atención Primaria de las comunidades autónomas participantes. Los detalles de este estudio han sido descritos en otra publicación ${ }^{18}$. La encuesta se realizó vía web, y fue anónima y autoadministrada. En Navarra, se envió a todos los correos electrónicos de los médicos de familia, pediatras y personal de enfermería un primer mensaje con un enlace que les permitía acceder a la encuesta. La encuesta estuvo accesible un mes, durante el cual se enviaron mensajes recordatorios cada 10 días a aquellos profesionales que aún no habían accedido a la misma.

El cuestionario fue diseñado a partir del utilizado por Kraut y col $^{19}$ y fue probado en un estudio piloto inicial. El cuestionario constaba de tres grandes bloques de preguntas. El primer bloque recopiló información acerca de la vacunación antigripal de los profesionales sanitarios en las temporadas 2008-09 a 2011-12, así como información sobre si ellos tenían alguna enfermedad con indicación de la vacunación antigripal. El segundo bloque indagó sobre las actitudes y percepciones frente a la vacunación antigripal, evaluadas en una escala tipo Likert. Las preguntas incluyeron la motivación por vacunarse, la preocupación por contagiarse en el trabajo o por enfermar, la preocupación por contagiar a la familia o a sus pacientes, la percepción de que la gripe pueda ser una enfermedad grave, y si consideraba que la vacuna es efectiva frente a la gripe y sus complicaciones. El tercer bloque de preguntas recogió datos sociodemográficos y profesionales que incluyeron el sexo, el grupo de edad (25-34, 35-44, 45-54 y $\geq 55$ años), si convivía con niños $<2$ años o de 2-15 años, si tenía convivientes $\geq 65$ años o con enfermedades crónicas, la categoría profesional (médico 
de familia, pediatra o personal de enfermería) y si participaba en la red centinela de vigilancia de la gripe.

Las respuestas relativas a las actitudes y percepciones frente a la vacunación antigripal fueron agrupadas en dos categorías: positivas (totalmente de acuerdo y bastante de acuerdo) y negativas (ni de acuerdo ni en desacuerdo, bastante en desacuerdo $\mathrm{y}$ totalmente en desacuerdo).

El proyecto fue aprobado por el Comité de Ética e Investigación del Instituto de Investigación en Atención Primaria Jordi Gol.

\section{Análisis estadístico}

Se evaluaron los factores asociados a la vacunación antigripal de los profesionales sanitarios durante la temporada 2011-12, incluyendo sus actitudes y percepciones ante la gripe y la vacuna.

Se utilizó la prueba Chi-cuadrado para comparar las proporciones. Se analizó la tendencia de la vacunación durante las temporadas 2008-09 a 2011-12 mediante el test de Chi-cuadrado de tendencia lineal. Se realizaron análisis bivariantes y multivariantes de regresión logística para estimar las odds ratios (OR) con sus intervalos de confianza (IC) al 95\%. Los modelos de regresión logística multivariantes fueron ajustados por las variables sociodemográficas y de tipo profesional que se mencionaron en el tercer bloque de preguntas de la encuesta. El paquete estadístico utilizado fue el SPSS 20.0.

\section{RESULTADOS}

\section{Cobertura de la vacuna antigripal durante la temporada 2011-12}

Se envió la encuesta a los 1.083 profesionales de atención primaria de Navarra, de los cuales 534 (49\%) contestaron a la primera parte de la encuesta, que contenía el antecedente de vacunación antigripal en las últimas temporadas, y 381 (35\%) finalizaron la encuesta.

De los 534 que aportaron información sobre su antecedente de vacunación an- tigripal, el 60,9\% habían recibido la vacuna estacional en la temporada 2008-09, el $61,2 \%$ en la temporada $2009-10$, y este porcentaje descendió al 58,4\% en la temporada 2010-11 y al 56,2\% en la temporada 2011-12, aunque esta tendencia no alcanzó significación estadística ( $\mathrm{p}=0,094)$. El 48,7\% se habían vacunado frente a la gripe pandémica A(H1N1)pdm09 en la temporada 2009-10. En los 381 profesionales que finalizaron la encuesta las coberturas de vacunación fueron superiores a las de los que no la finalizaron, pero la tendencia temporal fue similar (Tabla 1).

El grupo de profesionales que finalizaron la encuesta quedó conformado por 299 $(78,5 \%)$ mujeres y $82(21,5 \%)$ hombres. El $45,5 \%$ tenía una edad comprendida entre 45 y 54 años. El 54,3\% fueron profesionales de enfermería, $35,7 \%$ médicos de familia y $10,0 \%$ pediatras.

Durante la temporada 2011-12, el 59,1\% de los profesionales sanitarios que completaron la encuesta afirmó haberse vacunado frente a la gripe. Esta cobertura fue superior en los pediatras $(68,4 \%)$ y médicos de familia $(64,7 \%)$ que entre personal de enfermería $(53,6 \%)$; también fue mayor entre aquellos profesionales que tenían indicación de vacunación por enfermedad (73,5\%), entre los que tenían convivientes $\geq 65$ años o con enfermedades crónicas (67,3\%), y entre los que convivían con algún niño menor de 2 años (68,4\%) (Tabla 2).

El 33,1\% de los profesionales encuestados habían realizado actividades de formación sobre gripe y el $49,9 \%$ sobre vacunas (Tabla 2). El 14,4\% de los profesionales habían participado en actividades formativas sobre la gripe organizadas por los propios centros de salud y el $16,8 \%$ en actividades organizadas por la administración sanitaria. En cuanto a la formación sobre vacunaciones, el 18,1\% había participado en actividades organizadas por laboratorios farmacéuticos, el 16,8\% en actividades organizadas por sociedades científicas y colegios profesionales, el $16,0 \%$ en actividades organizadas por la administración sanitaria y el $12,6 \%$ en actividades organizadas por los centros de salud, no siendo opciones excluyentes. 
Tabla 1. Vacunación antigripal en los profesionales sanitarios de atención primaria de Navarra durante las temporadas 2008-09 a 2011-12

\begin{tabular}{|c|c|c|c|c|c|}
\hline & $\begin{array}{c}\text { Vacuna } \\
\text { estacional } \\
\text { temporada } \\
2008-09 \\
\text { N (\%) }\end{array}$ & $\begin{array}{c}\text { Vacuna } \\
\text { estacional } \\
\text { temporada } \\
2009-10 \\
\text { N (\%) }\end{array}$ & $\begin{array}{c}\text { Vacuna } \\
\text { pandémica } \\
\text { A(H1N1) } \\
\text { pdm09 } \\
\text { N (\%) }\end{array}$ & $\begin{array}{c}\text { Vacuna } \\
\text { estacional } \\
\text { temporada } \\
2010-11 \\
\text { N (\%) }\end{array}$ & $\begin{array}{c}\text { Vacuna } \\
\text { estacional } \\
\text { temporada } \\
2011-12 \\
\text { N (\%) }\end{array}$ \\
\hline Iniciaron la encuesta $(n=534)$ & $325(60,9)$ & $327(61,2)$ & $260(48,7)$ & $312(58,4)$ & $300(56,2)$ \\
\hline Interrumpieron la encuesta $(n=153)$ & $82(53,6)$ & $82(53,6)$ & $70(45,8)$ & $78(51,0)$ & $75(49,0)$ \\
\hline Completaron la encuesta $(\mathrm{n}=381)$ & $243(63,8)$ & $245(64,3)$ & $190(49,9)$ & $234(61,4)$ & $225(59,1)$ \\
\hline \multicolumn{6}{|c|}{ Profesionales que completaron la encuesta } \\
\hline \multicolumn{6}{|c|}{ Profesión } \\
\hline Médico de Familia & $102(75,0)$ & $98(72,1)$ & $83(61,0)$ & $95(69,9)$ & $88(64,7)$ \\
\hline Pediatra & $26(68,4)$ & $28(73,7)$ & $21(55,3)$ & $28(73,7)$ & $26(68,4)$ \\
\hline Enfermería & $115(55,6)$ & $119(57,5)$ & $86(41,5)$ & $111(53,6)$ & $111(53,6)$ \\
\hline \multicolumn{6}{|l|}{ Grupos de edad } \\
\hline 25-34 años & $10(34,5)$ & $11(37,9)$ & $11(37,9)$ & $15(51,7)$ & $16(55,2)$ \\
\hline 35-44 años & $53(60,9)$ & $50(57,5)$ & $37(42,5)$ & $43(49,4)$ & $47(54,0)$ \\
\hline 45-54 años & $115(66,5)$ & $121(69,9)$ & $87(50,3)$ & $113(65,3)$ & $103(59,5)$ \\
\hline$\geq 55$ años & $65(70,7)$ & $63(68,5)$ & $55(59,8)$ & $63(68,5)$ & $59(64,1)$ \\
\hline \multicolumn{6}{|l|}{ Sexo } \\
\hline Hombre & $60(73,2)$ & $56(68,3)$ & $49(59,8)$ & $54(65,9)$ & $53(64,6)$ \\
\hline Mujer & $183(61,2)$ & $189(63,2)$ & $141(47,2)$ & $180(60,2)$ & $172(57,5)$ \\
\hline
\end{tabular}

Tabla 2. Factores asociados a la vacunación antigripal de los profesionales sanitarios de atención primaria durante la temporada 2011-12

\begin{tabular}{|c|c|c|c|c|c|c|}
\hline Características & $\begin{array}{l}\text { Total } \\
\text { N (\%) }\end{array}$ & $\begin{array}{l}\text { Vacunados } \\
\mathrm{N}(\%)\end{array}$ & $\begin{array}{l}\text { Odds Ratio crudo } \\
\text { (IC95\%) }\end{array}$ & $\mathbf{p}$ & $\begin{array}{l}\text { Odds Ratio ajustado* } \\
\text { (IC95\%) }\end{array}$ & $\mathbf{p}$ \\
\hline \multicolumn{7}{|l|}{ Profesión } \\
\hline Médico de Familia & $136(35,7)$ & $88(64,7)$ & 1 & & 1 & \\
\hline Pediatra & $38(10,0)$ & $26(68,4)$ & $1,18(0,55-2,55)$ & 0,670 & $1,04(0,45-2,38)$ & 0,926 \\
\hline Enfermería & $207(54,3)$ & $111(53,6)$ & $0,63(0,40-0,98)$ & 0,042 & $0,62(0,36-1,07)$ & 0,088 \\
\hline \multicolumn{7}{|l|}{ Grupos de edad } \\
\hline 25-34 años & $29(7,6)$ & $16(55,2)$ & 1 & & 1 & \\
\hline 35-44 años & $87(22,8)$ & $47(54,0)$ & $0,96(0,41-2,22)$ & 0,914 & $0,87(0,35-2,16)$ & 0,760 \\
\hline 45-54 años & $173(45,4)$ & $103(59,5)$ & $1,20(0,54-2,64)$ & 0,659 & $1,17(0,50-2,75)$ & 0,715 \\
\hline$\geq 55$ años & $92(24,1)$ & $59(64,1)$ & $1,45(0,62-3,39)$ & 0,387 & $1,40(0,56-3,43)$ & 0,489 \\
\hline \multicolumn{7}{|l|}{ Sexo } \\
\hline Hombre & $82(21,5)$ & $53(64,6)$ & 1 & & 1 & \\
\hline Mujer & $299(78,5)$ & $172(57,5)$ & $0,74(0,45-1,23)$ & 0,247 & $0,95(0,52-1,75)$ & 0,872 \\
\hline \multicolumn{7}{|c|}{ Participación en la red centinela de gripe } \\
\hline No & $319(83,7)$ & $186(58,3)$ & 1 & & 1 & \\
\hline Sí & $62(16,3)$ & $39(62,9)$ & $1,21(0,69-2,13)$ & 0,501 & $0,94(0,50-1,75)$ & 0,839 \\
\hline \multicolumn{7}{|c|}{ Indicación de vacuna por enfermedad } \\
\hline No & $347(91,1)$ & $200(57,6)$ & 1 & & 1 & \\
\hline Sí & $34(8,9)$ & $25(73,5)$ & $2,04(0,93-4,50)$ & 0,077 & $1,95(0,87-4,38)$ & 0,107 \\
\hline \multicolumn{7}{|c|}{ Convivientes $\geq 65$ años o con enfermedades crónicas } \\
\hline No & $283(74,3)$ & $159(56,2)$ & 1 & & 1 & \\
\hline Sí & $98(25,7)$ & $66(67,3)$ & $1,61(0,99-2,61)$ & 0,054 & $1,72(1,05-2,84)$ & 0,033 \\
\hline \multicolumn{7}{|l|}{ Convivientes $<2$ años } \\
\hline No & $362(95,0)$ & $212(58,6)$ & 1 & & 1 & \\
\hline Sí & $19(5,0)$ & $13(68,4)$ & $1,53(0,57-4,12)$ & 0,397 & $1,80(0,63-5,21)$ & 0,275 \\
\hline \multicolumn{7}{|l|}{ Convivientes 2-15 años } \\
\hline No & $238(62,5)$ & $139(58,4)$ & 1 & & 1 & \\
\hline Sí & $143(37,5)$ & $86(60,1)$ & $1,08(0,70-1,64)$ & 0,739 & $1,16(0,71-1,90)$ & 0,545 \\
\hline \multicolumn{7}{|l|}{ Formación sobre gripe } \\
\hline No & $255(66,9)$ & $147(57,6)$ & 1 & & 1 & \\
\hline Sí & $126(33,1)$ & $78(61,9)$ & $1,19(0,77-1,85)$ & 0,427 & $1,03(0,62-1,70)$ & 0,916 \\
\hline \multicolumn{7}{|c|}{ Formación sobre vacunas } \\
\hline No & $191(50,1)$ & $112(58,6)$ & 1 & & 1 & \\
\hline Sí & $190(49,9)$ & $113(59,5)$ & $1,04(0,69-1,56)$ & 0,868 & $1,11(0,69-1,79)$ & 0,678 \\
\hline Total & $381(100)$ & $225(59,1)$ & & & & \\
\hline
\end{tabular}

* Análisis de regresión logística ajustado por las variables de la tabla. 
En el análisis multivariante de regresión logística, el único factor que mostró una asociación significativa con la vacunación de los profesionales fue el tener convivientes $\geq 65$ años o con enfermedades crónicas (OR: 1,72; IC 95\%: 1,05-2,84; p=0,033). Cuando el propio profesional presentaba alguna enfermedad crónica con indicación de vacunación antigripal la vacunación fue más frecuente, aunque el escaso número de profesionales en esta situación no permitió alcanzar significación estadística (OR: 1,95; IC 95\%: 0,87-4,38; $p=0,107)$. El haber realizado actividades de formación, tanto sobre gripe como acerca de vacunas, no se asoció a la vacunación, así como ninguna otra de las variables analizadas (Tabla 2).

En un análisis alternativo, se incluyó en el modelo multivariante anterior la vacunación antigripal en temporadas previas. La vacunación antigripal en la temporada 2011-12 mostró asociación con el haber recibido la vacuna pandémica (OR: 2,73; IC 95\%: $1,49-4,95 ; \mathrm{p}=0,001)$ y con el haber recibido alguna de las vacunas estacionales en las tres temporadas previas (OR: 20,87; IC 95\%: 8,98-48,48; $\mathrm{p}<0,001)$.

\section{Actitudes y percepciones de los profesionales de atención primaria ante su propia vacunación antigripal}

De los 381 profesionales que finalizaron la encuesta, el 59,6\% reconocieron que la gripe puede ser una enfermedad grave, y el $86,4 \%$ que la vacuna es efectiva para prevenir la gripe y sus complicaciones. El 38,3\% refirieron que su médico o unidad de salud laboral les había recomendado la vacunación.

Curiosamente el $22,3 \%$ de los profesionales que no habían recibido la vacuna antigripal en la temporada 2011-12 consideraron que la gripe puede ser una enfermedad grave y el $31,2 \%$ pensó que la vacuna es efectiva para prevenir la gripe y sus complicaciones. Sin embargo, el 22,3\% afirmó que varios de sus compañeros de trabajo no se habían vacunado y cerca del $18 \%$ prefería la inmunidad natural a la adquirida por la vacunación (Fig. 1).

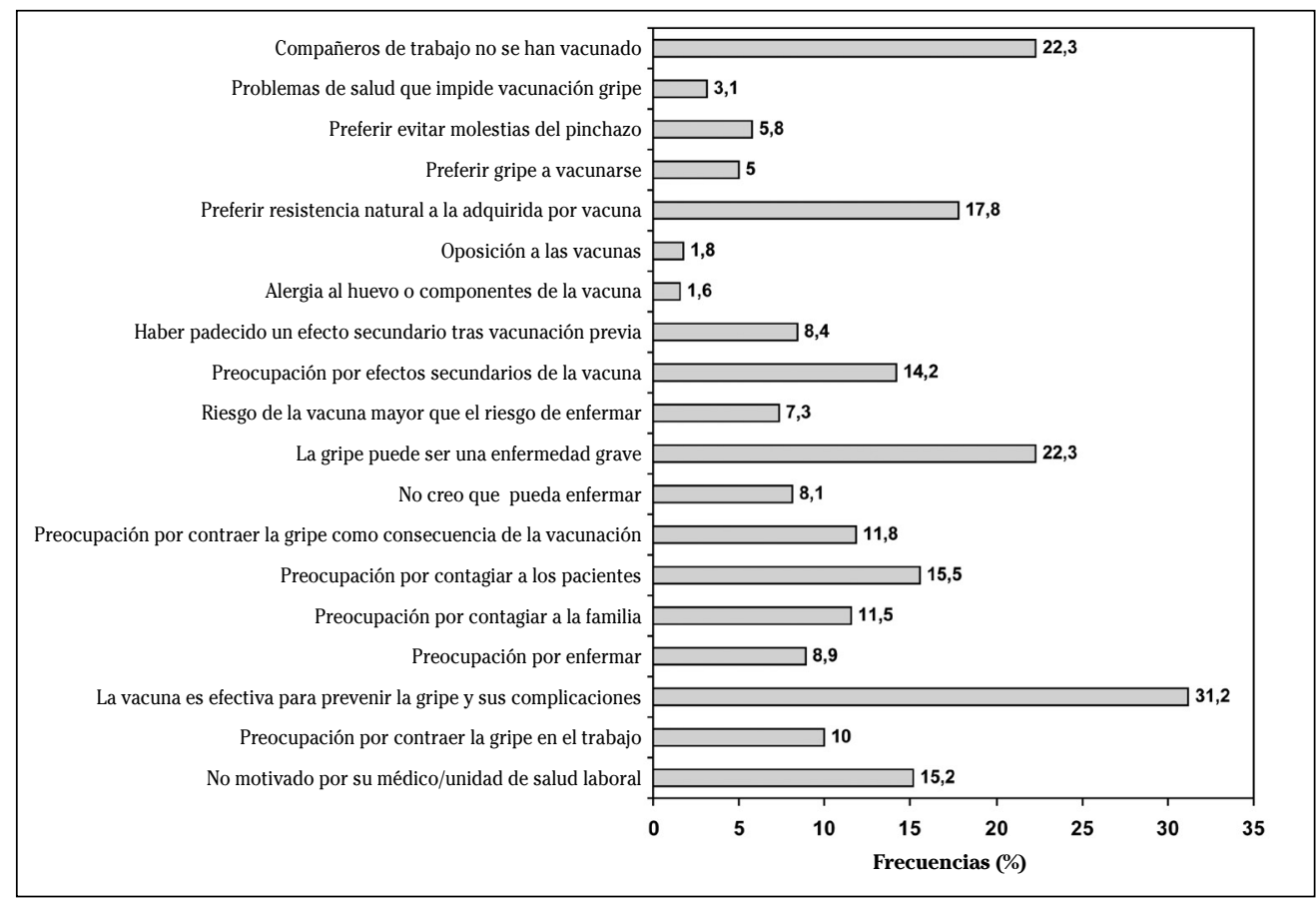

Figura 1. Actitudes y percepciones frente a la vacunación antigripal en los profesionales sanitarios que no se vacunaron frente a la gripe durante la temporada 2011-12 en Navarra. 
En los análisis bivariantes, el convencimiento de que la vacuna es efectiva, la preocupación por enfermar, por contagiarse en el trabajo y por contagiar a familiares o a los pacientes, se asociaron a una mayor probabilidad de vacunación de los profesionales. Sin embargo, el fuerte solapamiento entre muchas de estas percepciones hizo que al incluirlas en el análisis multivariante, solo mantuvieran asociación estadísticamente significativa e independiente con la vacunación entre los profesionales la preocupación por enfermar ellos mismos (OR: 4,59; IC 95\%: 2,30-9,15; $\mathrm{p}<0,001$ ) y por contagiar a sus pacientes (OR: 6,29; IC 95\%: 3,02-13,13; $\mathrm{p}<0,001$ ) (Tabla 3).

Tabla 3. Asociación entre las actitudes y percepciones de los profesionales sanitarios de atención primaria sobre la gripe y su vacunación antigripal en la temporada 2011-12

\begin{tabular}{|c|c|c|c|c|c|c|}
\hline Actitudes y percepciones & $\begin{array}{c}\text { Total } \\
\mathbf{N}\end{array}$ & $\begin{array}{l}\text { Vacunados } \\
\text { N (\%) }\end{array}$ & $\begin{array}{l}\text { Odds Ratio crudo } \\
\text { (IC95\%) }\end{array}$ & p & $\begin{array}{c}\text { Odss Ratio } \\
\text { ajustado* (IC95\%) }\end{array}$ & $\mathbf{p}$ \\
\hline \multicolumn{7}{|c|}{ Recomendación de la vacuna por el médico de cabecera o de salud laboral } \\
\hline No & 235 & $137(58,3)$ & 1 & & 1 & \\
\hline Sí & 146 & $88(60,3)$ & $1,09(0,71-1,65)$ & 0,703 & $0,63(0,36-1,13)$ & 0,119 \\
\hline \multicolumn{7}{|c|}{ Convencimiento de que la vacuna es efectiva } \\
\hline No & 52 & $15(28,8)$ & 1 & & 1 & \\
\hline Sí & 329 & $210(63,8)$ & $4,35(2,29-8,26)$ & $<0,001$ & $1,92(0,85-4,31)$ & 0,115 \\
\hline \multicolumn{7}{|c|}{ Preocupación por contagiarse en el trabajo } \\
\hline No & 178 & $60(33,7)$ & 1 & & 1 & \\
\hline Sí & 203 & $165(81,3)$ & $8,54(5,34-13,66)$ & $<0,001$ & $1,81(0,91-3,63)$ & 0,093 \\
\hline \multicolumn{7}{|l|}{ Preocupación por enfermar } \\
\hline No & 181 & $59(32,6)$ & 1 & & 1 & \\
\hline Sí & 200 & $166(83,0)$ & $10,10(6,23-16,36)$ & $<0,001$ & $4,59(2,30-9,15)$ & $<0,001$ \\
\hline \multicolumn{7}{|c|}{ Preocupación por contagiar a la familia } \\
\hline No & 163 & $51(31,3)$ & 1 & & 1 & \\
\hline Sí & 218 & $174(79,8)$ & $8,68(5,44-13,87)$ & $<0,001$ & $1,18(0,55-2,54)$ & 0,668 \\
\hline \multicolumn{7}{|c|}{ Preocupación por contagiar a pacientes } \\
\hline No & 129 & $32(24,8)$ & 1 & & 1 & \\
\hline Sí & 252 & $193(76,6)$ & $9,92(6,05-16,26)$ & $<0,001$ & $6,29(3,02-13,13)$ & $<0,001$ \\
\hline \multicolumn{7}{|c|}{ La gripe puede ser una enfermedad grave } \\
\hline No & 154 & $83(53,9)$ & 1 & & 1 & \\
\hline Sí & 227 & $142(62,6)$ & $1,43(0,94-2,17)$ & 0,092 & $0,70(0,40-1,25)$ & 0,234 \\
\hline
\end{tabular}

* Análisis de regresión logística ajustado por profesión, grupos de edad, sexo, participación como médico centinela, indicación de vacunación por enfermedad, convivientes $\geq 65$ años o con enfermedades crónicas, convivientes $<2$ años, convivientes entre 2 y 15 años, y las variables de la tabla.

\section{Actitudes de los profesionales de atención primaria ante la vacunación antigripal de sus pacientes}

La mayor parte de los profesionales sanitarios reconoció que la vacunación es la medida más importante para prevenir la gripe (82,2\%). El 75,3\% consideró que la vacunación del personal sanitario es importante para proteger a los pacientes, y el 95,8\% de los profesionales afirmó que vacunar a personas de alto riesgo cada temporada gripal es una medida efectiva para reducir las complicaciones (Tabla 4). 
Tabla 4. Opiniones y conocimientos de los profesionales sanitarios de atención primaria (n=381) sobre la vacuna de la gripe

\begin{tabular}{lcc}
\hline & N & \% \\
\hline La vacunación del personal sanitario es importante para proteger al paciente & 287 & 75,3 \\
La vacunación reduce los costes relacionados al tratamiento de la gripe & 289 & 75,9 \\
Vacunar a personas con alto riesgo reduce las complicaciones & 365 & 95,8 \\
Vacunar al personal sanitario reduce el riesgo de brotes porque aumenta la inmunidad de & 211 & 55,4 \\
grupo en lugares cerrados & 313 & 82,2 \\
Vacunar es la medida más importante para prevenir la gripe & \\
\hline
\end{tabular}

Por ello, la gran mayoría de los médicos de familia aconsejaron la vacunación antigripal a mayores de 65 años $(98,5 \%)$, a pacientes con enfermedades crónicas $(98,5 \%)$, pacientes inmunodeprimidos $(93,8 \%)$ y durante el segundo o tercer trimestre del embarazo (86,8\%). Sin embargo, se encontraron niveles más bajos de recomendación de la vacuna antigripal durante el primer trimestre del embarazo $(57,1 \%)$ y en el postparto (48,1\%) (Fig. 2).

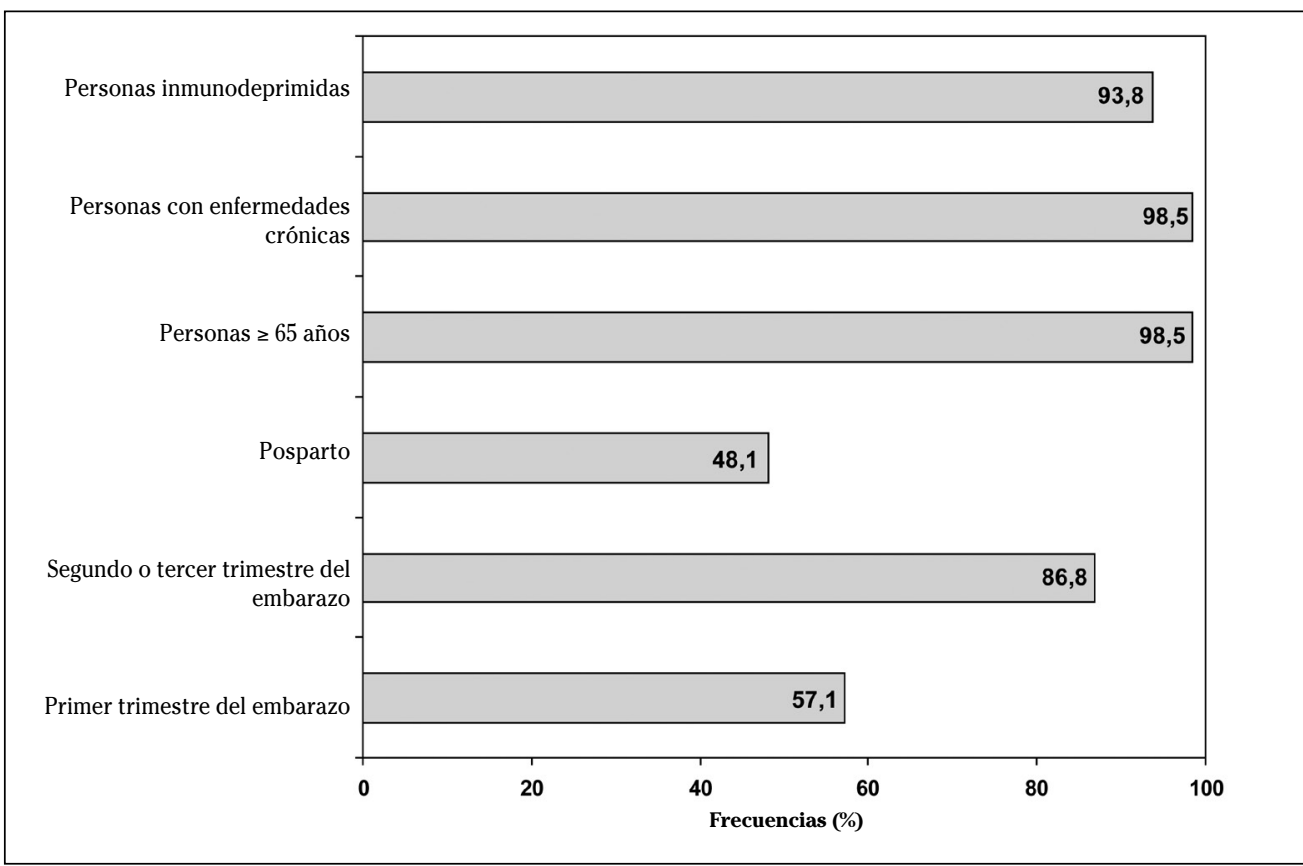

Figura 2. Recomendación de vacunación antigripal a los pacientes por parte de los médicos de familia.

\section{DISCUSIÓN}

El $56,2 \%$ de los profesionales sanitarios que iniciaron la encuesta refirieron haberse vacunado de la gripe estacional en la temporada 2011-12. Esta cobertura vacunal es todavía baja en comparación con los objetivos recomendados por la Organización Mundial de la Salud, pero es relativamente alta si la comparamos con las coberturas descritas por otros autores en profesiona- 
les sanitarios en España (20\%-50\%) $)^{10-12,20}$ y en otros países $(15 \%-40 \%)^{14,21,22}$.

Los resultados sobre la cobertura de vacunación antigripal representan al $49 \%$ de los profesionales sanitarios de atención primaria, que fueron los que respondieron a la primera parte de la encuesta, y es probable que los respondedores sean profesionales más preocupados por las cuestiones relacionadas con la gripe o las vacunas, o que tengan más facilidad en el uso de los sistemas informáticos. Por estos motivos, los resultados expuestos deben interpretarse con cautela.

La cobertura de vacunación antigripal entre los profesionales sanitarios de atención primaria en Navarra no ha sufrido cambios estadísticamente significativos durante el periodo estudiado. Tan solo, la vacunación pandémica tuvo coberturas inferiores, lo que puede explicarse porque la vacuna llegó cuando la onda gripal estaba muy avanzada y una parte de la población ya había padecido la gripe ${ }^{23}$. Algunos autores han descrito un cierto rechazo hacia la vacunación antigripal por parte de algunos profesionales sanitarios en las temporadas posteriores a la pandemia, lo que se ha mostrado en un descenso en las coberturas de vacunación antigripal ${ }^{12,24,25}$; sin embargo, este estudio no permite confirmar ni desmentir que pueda haber ocurrido algo similar entre los profesionales de atención primaria de Navarra. Entre los que respondieron, la actitud frente a la vacuna antigripal es positiva, pero no podemos generalizar esta observación a los profesionales que no respondieron a la encuesta.

Las coberturas fueron algo superiores entre los médicos de familia y pediatras que en el personal de enfermería, como han descrito en otro estudio ${ }^{12}$. El haber recibido la vacuna antigripal en alguna temporada previa, o convivir con alguna persona con enfermedad crónica o mayor de 65 años, se asoció con mayor probabilidad de vacunarse frente a la gripe. El sexo y la edad del profesional no conllevaron diferencias en su probabilidad de vacunarse, como ha sido descrito por otros autores ${ }^{16,26}$.

Un tercio de los profesionales habían realizado actividades de formación sobre gripe y la mitad sobre vacunas. Sin embargo, el haber realizado actividades formativas no se asoció a una mayor probabilidad de vacunación antigripal, lo que parece indicar que el aumento de conocimientos no implica necesariamente un cambio en las conductas. Los pediatras fueron los que habían recibido más formación sobre vacunas. Los principales promotores de las actividades formativas sobre la gripe fueron la administración sanitaria y los propios centros de salud. La iniciativa de las actividades formativas sobre vacunaciones estuvo repartida en proporciones parecidas entre los laboratorios farmacéuticos, las sociedades científicas o colegios profesionales, la administración sanitaria y los centros de salud.

La preocupación por enfermar o por contagiar a sus pacientes se asoció con mayor probabilidad de vacunación entre los trabajadores sanitarios, factores que también se describen en otros estudios ${ }^{27,28}$. La sensibilización de los trabajadores sanitarios acerca del riesgo de que puedan actuar de puente para la transmisión de la gripe entre el medio laboral y el entorno familiar parece tener un fuerte efecto motivador para su vacunación.

Una proporción considerable de los profesionales sanitarios reconocen que la gripe puede ser una enfermedad grave y que la vacuna es efectiva para prevenir la gripe y sus complicaciones, sin embargo, incluso entre estos profesionales la vacunación no es sistemática. La gran mayoría de los profesionales tienen claro que la vacunación a personas de alto riesgo es importante para reducir las complicaciones como se ha descrito en otro estudio ${ }^{28}$, y lo llevan a la práctica recomendando la vacuna antigripal entre los principales grupos de riesgo de la población general.

Varios autores han descrito diferentes estrategias para mejorar las coberturas en la vacunación antigripal entre los trabajadores sanitarios, como las campañas de información para destacar los beneficios de la vacuna, la vacunación del personal de altos cargos o solicitar una declaración de declinación entre los profesionales sanitarios que se niegan a recibir la vacuna ${ }^{29}$. 
McLennan y Wicker sugieren hacer obligatoria la vacunación antigripal del personal sanitario que atiende a pacientes más vulnerables, y solicitar la declaración y reubicar a los trabajadores no dispuestos a vacunarse $^{30}$. Por otra parte, hay experiencias exitosas de campañas de vacunación activa promoviendo la comunicación con los profesionales y ofreciendo algún tipo de incentivo ${ }^{31}$.

El principal predictor de la vacunación antigripal fue la vacunación en temporadas previas, lo que demuestra que aquellos profesionales que se han vacunado en alguna ocasión es muy probable que sigan haciéndolo. Esto permite definir dos grupos de profesionales en los que incidir de forma diferente: aquellos que se han vacunado anteriormente, en los que el objetivo es conseguir una mayor adherencia a la vacunación antigripal en sucesivas temporadas, y aquellos que nunca se han vacunado, en los que el objetivo ha de ser el romper las barreras y las inercias.

El presente estudio puede estar afectado por algunas limitaciones que han de tenerse en cuenta al interpretar los resultados. La tasa de respuesta a la encuesta fue baja, pero fue superior a la lograda en otro estudio basado en encuestas vía web en profesionales sanitarios ${ }^{19}$. Entre los profesionales que finalizaron la encuesta, la cobertura de vacunación frente a la gripe en la temporada 2011-12 fue algo mayor que la de los que abandonaron la encuesta, lo que demuestra la existencia de un sesgo de selección de profesionales más motivados por la vacunación. Un sesgo de características similares podría establecer diferencias entre los que iniciaron la encuesta con respecto a los que no la iniciaron, aunque no tenemos datos para poder evaluarlo. La mayoría de los profesionales que iniciaron la encuesta contestaron a todas las preguntas, lo que apoya la validez de la información recogida. El estado de vacunación durante cada temporada fue autorreferido por cada uno de los profesionales, por lo que podría estar afectado por un posible sesgo de recuerdo, aunque al tratarse de profesionales sanitarios y de los últimos cuatro años, es posible que este sesgo sea poco importante. Llupià y col encuentran una sobreestimación de la cobertura en profesionales sanitarios cuando se analizan datos autorreferidos con respecto a datos de vacunas registradas ${ }^{32}$.

En conclusión, la cobertura de vacunación antigripal entre los profesionales sanitarios de atención primaria en Navarra es relativamente alta, pero todavía está por debajo de los niveles deseables. Esto contrasta con el buen nivel de conocimientos y la actitud positiva de los profesionales hacia la vacunación antigripal. En la práctica, los factores que marcan una mayor cobertura de vacunación antigripal en los profesionales de atención primaria son la preocupación por enfermar, la preocupación por contagiar a los pacientes y el convivir con personas $\geq 65$ años o con enfermedades crónicas. Estos resultados han de tenerse en cuenta para diseñar futuras campañas de vacunación antigripal en profesionales sanitarios, tratando de recabar una mayor implicación de los mismos en la estrategia de prevención de la gripe, fomentando la adherencia a la vacunación entre los profesionales que se han vacunado en temporadas previas y tratando de atraer a los que normalmente no se vacunan.

\section{Agradecimientos}

Agradecemos la colaboración a todos los profesionales sanitarios de atención primaria de Navarra que participaron en la encuesta, y a la Dirección de Atención Primaria por su implicación para la organización de este estudio.

\section{BIBLIOGRAFÍA}

1. GLEZEN WP. Serious morbidity and mortality associated with influenza epidemics. Epidemiol Rev 1982; 4: 25-44.

2. Fifty-sixth World Health Assembly. Prevention and control of influenza pandemics and annual epidemics.2003. WHA56.19. Disponible en: http://apps.who.int/gb/archive/ pdf_files/WHA56/ea56r19.pdf.

3. Vanhems P, Voirin N, Roche S, Escuret V, Regis C, Gorain $\mathrm{C}$ et al. Risk of influenza-like illness in an acute health care setting during commu- 
nity influenza epidemics in 2004-2005, 20052006, and 2006-2007: a prospective study. Arch Intern Med 2011; 171: 151-157.

4. JoRdAn R, HAYWARD A. Should healthcare workers have the swine flu vaccine? BMJ 2009; 339: b3398.

5. Carman WF, Elder AG, Wallace la, McAulay K, WALKER A, MurRAY GD et al. Effects of influenza vaccination of health-care workers on mortality of elderly people in long-term care: a randomised controlled trial. Lancet 2000; 355: 93-97.

6. Potter J, Stott DJ, Roberts MA, Elder AG, O’Donnell B, KNight PV et al. Influenza vaccination of health care workers in long-termcare hospitals reduces the mortality of elderly patients. J Infect Dis 1997; 175: 1-6.

7. Castilla J, Godoy P, Domínguez A, Martín V, Delgado-Rodríguez M, Martínez-Baz I et al. Risk factors and effectiveness of preventive measures against influenza in the community. Influenza Other Respi Viruses 2013; 7: 177-183.

8. European Union. Council Recommendation of 22 December 2009 on seasonal influenza vaccination. Official Journal of the European Union L348:71-2. 2009. Disponible en: http:// eur-lex.europa.eu/LexUriServ/LexUriServ.do ?uri=OJ:L:2009:348:0071:0072:EN:PDF

9. Advisory Committee on Immunization Practices; Centers for Disease Control and Prevention (CDC). Immunization of health-care personnel: recommendations of the Advisory Committee on Immunization Practices (ACIP). MMWR Recomm Rep 2011; 60 (RR-7): $1-45$.

10. Jiménez-García R, Hernández-Barrera V, Carrasco-Garrido P, López de Andrés A, Pérez N, dE Miguel AG. Influenza vaccination coverages among children, adults, health care workers and immigrants in Spain: related factors and trends, 2003-2006. J Infect 2008; 57: 472-480.

11. Vírseda S, Restrepo MA, Arranz E, Magán-Tapia P, Fernández-Ruiz M, de La CÁmara AG et al. Seasonal and Pandemic A (H1N1) 2009 influenza vaccination coverage and attitudes among health-care workers in a Spanish University Hospital. Vaccine 2010; 28: 4751-4757.

12. SÁnchez-Payá J, Hernández-García I, García-Román V, CAMargo-Angeles R, BarrenengoA-SAÑUdo J, Villanueva-Ruiz $\mathrm{CO}$ et al. Influenza vaccination among healthcare personnel after pandemic influenza H1N1. Vaccine 2012; 30: 911915.

13. Bali NK, Ashraf M, Ahmad F, Khan UH, WiddowSON MA, LAL RB et al. Knowledge, attitude, and practices about the seasonal influenza vaccination among healthcare workers in Srinagar, India. Influenza Other Respi Viruses 2012. doi: 10.1111/j.1750-2659.2012.00416.x.

14. Maltezou hC, Maragos A, Katerelos P, Paisi A, Karageorgou K, Papadimitriou T et al. Influenza vaccination acceptance among health-care workers: a nationwide survey. Vaccine 2008; 26: 1408-1410.

15. Brandt C, Rabenau HF, Bornmann S, Gottschalk R, WiCKER S. The impact of the 2009 influenza A (H1N1) pandemic on attitudes of healthcare workers toward seasonal influenza vaccination 2010/11. Euro Surveill 2011; 16. pii: 19854.

16. Hothersall EJ, de Bellis-Ayres S, Jordan R. Factors associated with uptake of pandemic influenza vaccine among general practitioners and practice nurses in Shropshire, UK. Prim Care Respi J 2012; 21: 302-307.

17. Ward K, Seale H, Zwar N, Leask J, Macintyre CR. Annual influenza vaccination: coverage and attitudes of primary care staff in Australia. Influenza Other Respi Viruses 2011; 5: 135141.

18. Baricot M, Toledo D, Castilla J, Torner N, Godoy $\mathrm{P}$, Astray J et al. Estudio de actitudes y conocimientos sobre la vacunación antigripal en personal sanitario de atención primaria. Temporada 2011-2012. Vacunas 2013; 14: 22-29.

19. Kraut A, Graff L, McLean D. Behavioral change with influenza vaccination: factors influencing increased uptake of the pandemic H1N1 versus seasonal influenza vaccine in health care personnel. Vaccine 2011; 29: 8657-8663.

20. JimÉnez-García R, Hernández-Barrera V, Carrasco-Garrido P, Sierra-Moros MJ, Martínez-HerNÁNDEZ D, DE MiguEL AG. Influenza vaccination coverages among spanish children, adults and health care workers. Infection 2006; 34 : 135-141.

21. Wicker S, Rabenau HF, Doerr HW, Allwinn R. Influenza vaccination compliance among health care workers in a german university hospital. Infection 2009; 37: 197-202.

22. Lindley MC, Yonek J, Ahmed F, Perz JF, Williams Torres G. Measurement of influenza vaccination coverage among healthcare personnel in US hospitals. Infect Control Hosp Epidemiol 2009; 30: 1150-1157.

23. Castilla J, Morán J, Fernández-Alonso M, MartínEZ Artola V, Zamora MJ, Mazón A et al. Caracterización de la pandemia de gripe A H1N1 2009 en Navarra. An Sist Sanit Navar 2010; 33: $287-295$ 
24. Vaux S, Van Cauteren D, Guthmann JP, Le Strat $\mathrm{Y}$, VAILLANT V, DE VALK $\mathrm{H}$ et al. Influenza vaccination coverage against seasonal and pandemic influenza and their determinants in France: a cross-sectional survey. BMC Public Health $2011 ; 11: 30$.

25. Arda B, Durusoy R, Yamazhan T, Sipahi OR, Ta baKan M, Pullukçu $\mathrm{H}$ et al. Did the pandemic have an impact on influenza vaccination attitude? A survey among health care workers. BMC Infect Dis 2011; 11: 87.

26. Chen SC, Hawkins G, Aspinall E, Patel N. Factors influencing uptake of influenza A (H1N1) vaccine amongst healthcare workers in a regional pediatric centre: lessons for improving vaccination rates. Vaccine 2012; 30: 493-497.

27. Martinello RA, Jones L, Topal JE. Correlation between healthcare workers' knowledge of influenza vaccine and vaccine receipt. Infect Control Hosp Epidemiol 2003; 24: 845-847.
28. Hollmeyer HG, Hayden F, Poland G, Buchholz U. Influenza vaccination of health care workers in hospitals-a review of studies on attitudes and predictors. Vaccine 2009; 27: 3935-3944.

29. Picazo JJ, González Romo F, Salleras Sanmartí L, Bayas Rodríguez JM, Alvarez Pasquín MJ. Encuesta sobre vacunación de adultos en España. Gripe y neumococo. Vacunas 2012; 13: 100-111.

30. McLennan S, Wicker S. Reflections on the influenza vaccination of healthcare workers. Vaccine 2010; 28: 8061-8064.

31. Llupià A, García-Basteiro AL, Olivé V, Costas L, Ríos J, Quesada S et AL. New interventions to increase influenza vaccination rates in health care workers. Am J Infect Control 2010; 38: 476-481.

32. Llupià A, García-Basteiro Al, Mena G, Ríos J, Puig $\mathrm{J}$, BAYAS JM et al. Vaccination behaviour influences self-report of influenza vaccination status: a cross-sectional study among health care workers. PLoS One 2012; 7: e39496. 